\title{
Raman Scattering from Frustrated Quantum Spin Chains
}

\author{
Wolfram Brenig \\ Institut für Theoretische Physik, Universität zu Köln Zülpicher Str. 77, 50937 Köln, Germany
}

(March 9, 2021)

\begin{abstract}
Magnetic Raman scattering from a frustrated spin-1/2 Heisenberg-chain is considered with a focus on the uniform phase of the spin-Peierls compound $\mathrm{CuGeO}_{3}$. The Raman intensity is analyzed in terms of a Loudon-Fleury scattering process using a spinless-fermion mean-field theory developed for the frustrated spin-chain. A comparison to experimental data is presented and the frustration and temperature dependence is studied. In good agreement with observed spectra a broad inelastic four-spinon continuum is found at low temperatures above the spin-Peierls transition. At high temperatures the intensity develops a quasi-elastic line analogous to experiment.
\end{abstract}

PACS: 75.10.Jm, 75.40.Gb, 78.30.-j 


\section{INTRODUCTION}

Elementary excitations of one-dimensional (1D) quantum spin-chains exhibit a number of remarkable subtleties, such as the magnon continuum of the isotropic $1 \mathrm{D}$ spin-1/2 Heisenberg model or the excitation-gap for integer-spin systems as conjectured by Haldane. Recent and extensive studies of 'spin-ladder' compounds as well as of the germanate $\mathrm{CuGeO}_{3}$ [四] have lead to renewed interest in quantum spin-chains. In this context, magnetic Raman scattering, if symmetry-allowed, has developed into an important tool to investigate the local spin dynamics [2] Here I will establish a simple framework to interpret the magnetic Raman scattering from a frustrated quantum spin-chain with a particular focus on the uniform phase of $\mathrm{CuGeO}_{3}$.

$\mathrm{CuGeO}_{3}$ is a quasi-1D anorganic spin-Peierls compound with a dimerization transition at a temperature $T_{S P} \simeq 14 K[1,5,6]$. Its structure comprises of weakly coupled $\mathrm{CuO}_{2}$ chains, with copper in a spin-1/2 state [7]. Since the nearest-neighbor (n.n.) exchange-coupling between copper spins along the $\mathrm{CuO}_{2}$ chains is strongly reduced by almost orthogonal intermediate oxygen states [8] the next-nearest-neighbor (n.n.n.) exchange is relevant. Both, n.n. and n.n.n. exchange, are antiferromagnetic [9] implying intra-chain frustration. A minimal model of $\mathrm{CuGeO}_{3}$ is the $J_{1}-J_{2}-\delta$ model

$$
H=J_{1} \sum_{l}\left[\left(1+(-)^{l} \delta\right) \mathbf{S}_{l} \cdot \mathbf{S}_{l+1}+\alpha \mathbf{S}_{l} \cdot \mathbf{S}_{l+2}\right]
$$

Here, $\mathbf{S}_{l}$ is a spin-1/2 operator, $J_{1} \approx 160 K$ 10 12 is the n.n. exchange-coupling constant and $\delta$ resembles the lattice dimerization which is finite for $T<T_{S P}$ only. $\alpha$ is the intrachain frustration-ratio $\alpha=J_{2} / J_{1}$ where $J_{2}$ is the n.n.n. exchange-coupling constant. A final consensus on the precise magnitude of $\alpha$ is still lacking. Studies of the magnetic susceptibility have resulted in $\alpha \approx 0.24$ 110 as well as in $\alpha \approx 0.35$ [11]. The latter is consistent with a comparative investigation of magnetic susceptibility and thermal expansion [12. Therefore, very likely, $\mathrm{CuGeO}_{3}$ displays a frustration induced contribution to the spingap at zero temperature irrespective of the actual lattice dimerization [13. Depending on 
the magnitude of the frustration the zero-temperature dimerization $\delta(T=0)$ has to be on the order of $\delta(0)=0.01 \ldots 0.03$ such as to enforce the size of the spin-gap observed in inelastic neutron scattering (INS) [5].

Magnetic excitations in $\mathrm{CuGeO}_{3}$ are clearly distinct among the uniform, i.e. $T>T_{S P}$, and the dimerized, i.e. $T<T_{S P}$, phase. While the dynamic structure factor exhibits a gapless two-spinon continuum similar to that of the 1D Heisenberg chain above $T_{S P}$ [14, well defined magnon-like excitations have been observed below $T_{S P}$ [15]. These magnons reside within the spin-gap and are split off from the two-spinon continuum. They have been interpreted as two-spinon bound states 16, 17.

Magnetic Raman scattering from $\mathrm{CuGeO}_{3}$ has been observed both, in the dimerized as well as in the uniform phase 24 . In the low temperature uniform phase, for $T_{S P}<T \ll J_{1}$, the Raman spectrum displays a broad continuum centered at $\hbar \omega \approx 2.4 J_{1}$. Early on this continuum has been related to four-spinon excitations [2 2 , 18]. In the high temperature uniform phase, and in addition to the four-spinon continuum, the spectra show a pronounced quasi-elastic line. In the dimerized phase the Raman intensity develops a gap at approximately 1.5-1.8 $\Delta_{S T}$ [20] where $\Delta_{S T}$ is the singlet-triplet gap observed in INS [5]. Moreover, four characteristic peaks appear in the spectrum the lowest one of which at $30 \mathrm{~cm}^{-1}$ 'coincides' with the Raman gap. At present the interpretations of these peaks are controversial. Tentatively the $30 \mathrm{~cm}^{-1}$ line has been attributed to a continuum of two-magnon bound states [19,20]. A possible dimensional crossover effect has been invoked for the $225 \mathrm{~cm}^{-1}$ line [3,20]. In this respect the influence of inter-chain coupling in $\mathrm{CuGeO}_{3}$ is an open problem [21].

Here I will focus on Raman scattering from the uniform phase of the $J_{1}-J_{2}-\delta$ model, i.e. at $\delta=0$. First I will describe a spinless-fermion mean-field theory to treat the $J_{1}-J_{2}$ Hamiltonian. Next the Raman intensity is expressed in terms of a four-fermion correlation function. Finally results for the Raman spectra are compared with experimental findings and are contrasted against other theoretical approaches. 


\section{MEAN-FIELD THEORY OF THE $J_{1}-J_{2}$ MODEL}

The spinless-fermion mean-field (MF) theory for the $J_{1}-J_{2}$ model is based on the Jordan-Wigner (JW) representation [22] of the spin algebra, i.e. $S_{l}^{z}=\left(c_{l}^{\dagger} c_{l}-\frac{1}{2}\right)$ and $S_{l}^{+}=(-)^{l} \prod_{j<l}\left(1-c_{j}^{\dagger} c_{j}\right) c_{l}^{\dagger}$, where $c_{l}^{(\dagger)}$ are fermion operators. Inserting this into (11) at $\delta=0$ one obtains

$$
\begin{aligned}
& H=\sum_{l} {\left[-\frac{1}{2} c_{l}^{\dagger} c_{l+1}-\frac{1}{2} c_{l+1}^{\dagger} c_{l}-c_{l}^{\dagger} c_{l}+c_{l}^{\dagger} c_{l} c_{l+1}^{\dagger} c_{l+1}+\frac{1}{4}+\frac{\alpha}{2} c_{l}^{\dagger} c_{l+2}+\frac{\alpha}{2} c_{l+2}^{\dagger} c_{l}\right.} \\
&\left.+\alpha\left(-c_{l}^{\dagger} c_{l+2} c_{l+1}^{\dagger} c_{l+1}-c_{l+1}^{\dagger} c_{l+1} c_{l+2}^{\dagger} c_{l}-c_{l}^{\dagger} c_{l}+c_{l}^{\dagger} c_{l} c_{l+2}^{\dagger} c_{l+2}+\frac{1}{4}\right)\right]
\end{aligned}
$$

where $J_{1}$ is set to unity in section $\mathbb{\square}$ and $\mathbb{\Pi 1}$. $l$ runs over the lattice sites. The terms proportional to $\alpha$ are absent in the JW representation of the isotropic n.n. Heisenberg model. In contrast to the n.n. model, both, the transverse as well as the longitudinal n.n.n. exchange-interaction lead to four-fermion vertices. Longer range spin-exchange leads to even higher order couplings, as is obvious from the JW representation which implies $2 l-$ fermion vertices for $l$-th.-nearest-neighbor spin-exchange. To proceed I treat Hamiltonian (2) in MF-approximation. Allowing for all contractions of type $\left\langle c_{l}^{\dagger} c_{m}\right\rangle$ one gets

$$
\begin{aligned}
H_{M F}=\sum_{k}\{ & -[1+(A+B)(1-2 \alpha)] \cos (k) c_{k}^{\dagger} c_{k} \\
& \left.+\frac{i}{2}(A-B)(1+2 \alpha) \sin (k)\left(c_{k}^{\dagger} c_{k+\pi}-c_{k+\pi}^{\dagger} c_{k}\right)\right\}+ \text { const. },
\end{aligned}
$$

where $k$ is the momentum, $A=A^{\star}=\left\langle c_{2 l}^{\dagger} c_{2 l+1}\right\rangle$, and $B=B^{\star}=\left\langle c_{2 l+1}^{\dagger} c_{2 l}\right\rangle$. In principle

contractions of type $D=D^{\star}=\left\langle c_{2 l+2}^{\dagger} c_{2 l}\right\rangle$ do occur, however, their selfconsistent value can be shown to vanish identically. Note that (3) allows for both, a uniform, if $A-B=0$, and a gaped phase, if $A-B \neq 0$. Here no attempt will be made to describe the spindimer state using $A \neq B$ and the relevance of the gaped solution of the MF-theory will be discussed elsewhere. In the uniform case (3) resembles a single-band spinon gas with a hopping amplitude $t(T, \alpha)$ to be determined selfconsistently

$$
H_{M F}=\sum_{k} \epsilon_{k} c_{k}^{\dagger} c_{k}
$$




$$
\begin{aligned}
\epsilon_{k} & =-t(T, \alpha) \cos (k)=-[1+2 A(1-2 \alpha)] \cos (k) \\
A & =\frac{2}{N} \sum_{0 \leq k \leq \pi} \cos (k) f\left(\epsilon_{k}\right)
\end{aligned}
$$

where $f(\epsilon)=[\exp (\epsilon / T)+1]^{-1}$ is the Fermi function. At zero temperature the spinon dispersion simplifies to

$$
\epsilon_{k}(T=0)=-[1+2(1-2 \alpha) / \pi] \cos (k)
$$

For vanishing frustration this is identical to Bulaevskii's result $-(1+2 / \pi) \cos (k)$ [23]. The latter is known to compare reasonably well with the exact spinon dispersion $\epsilon_{k}=-\pi / 2 \cos (k)$ by des Cloizeaux and Pearson [24]. For finite $\alpha$ the MF-theory results in a frustration induced softening of the spinon stiffness which, for $T=0$, can be expressed as

$$
\frac{v_{s}(\alpha)}{v_{s}(0)}=1-\frac{4}{2+\pi} \alpha \approx 1-0.778 \alpha
$$

where $v_{s}(\alpha)=\partial \epsilon_{k} /\left.\partial k\right|_{k=\pi / 2}$ is the spinon velocity. Eqn. (6) is qualitatively consistent with a numerical study where $v_{s}(\alpha) / v_{s}(0) \approx 1-1.12 \alpha$ has been found [17. In fig. 1 $t(T, \alpha)$ is depicted as a function of temperature for various values of frustration. As is obvious the MF spinon-stiffness is decreased, both, as a function of increasing frustration and temperature. At zero temperature the MF ground state energy is given by $E_{0}(\alpha)=$ $N\left(-1 / \pi-1 / \pi^{2}+2 \alpha / \pi^{2}\right)$ which, for $\alpha=0$, leads to $E_{0}(0) \approx-0.420$ [23]. This agrees reasonably well with the Bethe-Ansatz result $E_{0}=1 / 4-\ln (2) \approx-0.443$. Moreover, the

linear frustration dependence of the MF ground state energy, $\left(E_{0}(\alpha)-E_{0}(0)\right) / N \approx 0.203 \alpha$ is close to that found in finite-chain diagonalization [25], where $\left(E_{0}(\alpha)-E_{0}(0)\right) / N \approx 0.177 \alpha$ for $\alpha \lesssim 0.3$.

\section{RAMAN SCATTERING}

The MF-theory is a convenient tool to study Raman scattering from the $J_{1}-J_{2}$ model. The Raman vertex is given by Loudon-Fleury's photon-induced super-exchange operator 26 


$$
R=\sum_{l m} T_{l m}\left(\mathbf{E}_{\text {in }} \cdot \mathbf{n}_{l m}\right)\left(\mathbf{E}_{\text {out }} \cdot \mathbf{n}_{l m}\right) \mathbf{S}_{l} \cdot \mathbf{S}_{m}
$$

Here $T_{l m}$ sets the coupling strength, $\mathbf{E}_{\text {in }}\left(\mathbf{E}_{\text {out }}\right)$ refers to the field of the in(out)going light, and $\mathbf{n}_{l m}$ labels a unit vector connecting the sites $l$ and $m$. By symmetry, in a strictly 1D situation, (17) leads to scattering only for parallel polarization along the c-directed chains. Since the real-space decay of $T_{l m}$ is comparable to that of the exchange integrals in (11) it is sufficient to consider a Raman operator with at most n.n.n. spin-exchange

$$
R=C \sum_{l}\left(\mathbf{S}_{l} \cdot \mathbf{S}_{l+1}+\beta \mathbf{S}_{l} \cdot \mathbf{S}_{l+2}\right)
$$

The scattering intensity $I(\omega)$ is obtained via the fluctuation dissipation theorem $I(\omega)=$ $\chi^{\prime \prime}(\omega) /\left(1-e^{-\omega / T}\right)$ from the dynamical susceptibility of the Raman operator

$$
\chi^{\prime \prime}(\omega)=\operatorname{Im}[\chi(\omega+i \eta)]=R e \int_{0}^{+\infty} d t e^{i(\omega+i \eta) t}\langle[R(t), R]\rangle
$$

where $\langle\ldots\rangle$ denotes the thermal average.

Frustration of unequal magnitude regarding the $J_{1}-J_{2}$ model and the Raman operator is mandatory for non-vanishing inelastic scattering. If $\alpha=\beta$ the Hamiltonian for $\delta=0$ and the Raman operator commute which leads to elastic scattering only. The inelastic intensity which results from (8) is identical to that of a Raman operator $R^{\prime}=R-\gamma H$. Setting $\gamma=C \beta / \alpha$ or $\gamma=C$ leads to scattering by a renormalized n.n. or n.n.n. Raman operator only

$$
R_{1}=C\left(1-\frac{\beta}{\alpha}\right) \sum_{l} \mathbf{S}_{l} \cdot \mathbf{S}_{l+1} \quad \text { or } \quad R_{2}=C(\beta-\alpha) \sum_{l} \mathbf{S}_{l} \cdot \mathbf{S}_{l+2}
$$

At present, an exact treatment of (9) is not feasible and the Raman intensity of any approximate evaluation will depend on $\gamma$. In this respect $R_{2}$ is the proper choice for $\alpha, \beta \ll 1$ since it guarantees that $I(\omega) \propto(\beta-\alpha)^{2}$. This is not obvious for $R_{1}$ or other values of $\gamma$. In those cases, even for $\alpha, \beta \rightarrow 0, R^{\prime}$ may contain a component of order unity, proportional to the Hamiltonian, which has to be projected out. To avoid this complication $R_{2}$ will be considered hereafter. 
Using the JW-fermions the Raman operator $R_{2}$ can be expressed in terms of a fourfermion operator

$$
\begin{aligned}
& R_{2}=C(\beta-\alpha) \sum_{k, k^{\prime}, q} h\left(k, k^{\prime}, q\right) c_{k}^{\dagger} c_{k+q} c_{k^{\prime}}^{\dagger} c_{k^{\prime}-q}+\Lambda_{1 p h} \\
& h\left(k, k^{\prime}, q\right)=\cos (2 q)-\cos (2 k+q)-\cos \left(2 k^{\prime}-q\right),
\end{aligned}
$$

where $\Lambda_{1 p h}$ labels all one-particle-hole excitations of the JW-transform of $R_{2}$. Since these occur at zero total momentum they do not contribute to inelastic Raman scattering within MF-theory [27]. Using (11), the Raman intensity (9) is written in terms of the four-fermion propagator

$$
\begin{aligned}
\chi(\tau)=C^{2}(\alpha-\beta)^{2} \sum_{k, k^{\prime}, q, p, p^{\prime}, r} & {\left[h\left(k, k^{\prime}, q\right) h\left(p, p^{\prime}, r\right)\right.} \\
& \left.\left\langle T_{\tau}\left(c_{k}^{\dagger}(\tau) c_{k+q}(\tau) c_{k^{\prime}}^{\dagger}(\tau) c_{k^{\prime}-q}(\tau) c_{p^{\prime}-r}^{\dagger} c_{p^{\prime}} c_{p+r}^{\dagger} c_{p}\right)\right\rangle\right] .
\end{aligned}
$$

Here $\tau$ is the imaginary time and $\chi^{\prime \prime}(\omega)$ results from the usual analytic continuation $\chi^{\prime \prime}(\omega)=$ $-\operatorname{Im}\left[\chi\left(i \omega_{n} \rightarrow \omega+i 0^{+}\right)\right]$where $\omega_{n}=2 n \pi T$ is a Bose Matsubara-frequency. On the level of $\mathrm{MF}$-theory the four-fermion propagator (12) is evaluated neglecting all vertex corrections and using the MF one-particle Green's functions corresponding to (4). After a number of standard manipulations I obtain

$$
\begin{aligned}
\chi_{M F}^{\prime \prime}(\omega)= & -\frac{1}{2 \pi^{2}} \int_{-\pi}^{\pi} d q \int_{-\pi}^{\pi} d k \sum_{k^{\prime}}\left\{\frac{g^{2}\left(k, k^{\prime}+q, q\right)}{\sqrt{[2 t \sin (q / 2)]^{2}-\left(\epsilon_{k+q}-\epsilon_{k}-\omega\right)^{2}}}\right. \\
& {\left.\left[f\left(\epsilon_{k}\right)-f\left(\epsilon_{k+q}\right)\right]\left[f\left(\epsilon_{k^{\prime}}\right)-f\left(\epsilon_{k^{\prime}+q}\right)\right]\left[n\left(\epsilon_{k^{\prime}+q}-\epsilon_{k^{\prime}}+\omega\right)-n\left(\epsilon_{k^{\prime}+q}-\epsilon_{k^{\prime}}\right)\right]\right\}, }
\end{aligned}
$$

where $g\left(k, k^{\prime}, q\right)=\frac{1}{2}\left[h\left(k, k^{\prime}, q\right)+h\left(k, k^{\prime}, k^{\prime}-k-q\right)\right]$ and the discrete sum on $k^{\prime}$ runs over the set of solutions of

$$
\left.\left.\sin \left(k^{\prime}+q / 2\right)=\frac{\epsilon_{k+q}-\epsilon_{k}-\omega}{2 t \sin (q / 2)} \quad ; \quad k^{\prime} \in\right]-\pi, \pi\right]
$$

This concludes the MF-theory of Raman scattering. 


\section{DISCUSSION}

In fig. 2 I compare the MF-intensity obtained from a numerical integration of (13) (solid and dashed lines) at $T=20 K$ and $12.8 K$, for $J_{1}=160 K$ and two values of frustration $\alpha=0.24$ and $\alpha=0.35$ with an experimental Raman spectrum observed at $T=20 K$ in the uniform phase of $\mathrm{CuGeO}_{3}$ [4, 18] (dashed--dotted line with markers). Phonon lines at $184 \mathrm{~cm}^{-1}$ and $330 \mathrm{~cm}^{-1}$ have been removed. This Raman data is consistent with other published results [2]:3]. The absolute magnitude of the observed Raman intensity as well as the coupling strength $C$ of the scattering operator (8) are unknown quantities. Therefore the two sets of MF-intensities for distinct $\alpha$ and the experimental spectrum have been normalized on a scale of arbitrary units. The theoretical parameters in fig. 2 represent no attempt at a 'best fit' to the data, rather they have been chosen among those obtained independently from studies of the magnetic susceptibility of $\mathrm{CuGeO}_{3}[10$ [12]. Figure 2 displays reasonable agreement between theory and experiment, although details of the experimental spectrum, i.e. the structure at 375 wave numbers and the initial curvature are not reproduced by the MF-theory. Evidently a value of $\alpha=0.24$ [10 is slightly more favored by this comparison at $J_{1}=160 \mathrm{~K}$ than $\alpha=0.35$ [11,12]. Similar findings have been made in numerical studies [20]. However, since the maximum of the MF-intensity roughly scales with the maximum of the spinon-dispersion $\propto J_{1}[1+2(1-2 \alpha) / \pi]$, a better agreement with $\alpha=0.35$ instead of $\alpha=0.24$ can also be reached simply by increasing $J_{1}$ by $\sim 10 \%$.

The comparison in fig. 2 strongly corroborates a study [18 of Raman scattering from the uniform phase of $\mathrm{CuGeO}_{3}$ which is based the 'solitonic' mean-field description of the XXZ Heisenberg chain by Gómez-Santos [28]. This approach employs a domain-wall representation of the spin chain which leads to formal developments very different from the straightforward application of the Jordan-Wigner type of MF-theory presented here.

In fig. 3 the temperature dependence of the MF-spectrum is shown, both, in terms of the intensity $I(\omega)$ - which is observed in experiment - and the Raman-operator susceptibility $\chi^{\prime \prime}(\omega)$. The latter exhibits a left-shift of its maximum upon increase of the temperature. 
This leads to enhanced low-frequency spectral weight which, by virtue of the Bose-prefactor, turns into a quasi-elastic line in $I(\omega)$ for $T \gtrsim J_{1}$. A corresponding tendency has been detected in finite-temperature Lanczos studies [32]. In MF-theory the high-temperature enhancement of the low-frequency spectral weight in $\chi^{\prime \prime}(\omega)$ is due to the reduction of the spinon-stiffness as a function of increasing temperature, see fig. 1, and due to the 'smearing' induced by the Fermi- and Bose-functions contained in (13). It is tempting to relate the behavior depicted in fig. 3 to the high-temperature quasi-elastic line observed in the uniform phase of $\mathrm{CuGeO}_{3}$ [2 - However, in $\mathrm{CuGeO}_{3}$ the four-spinon continuum is only weakly shifted by temperature and remains more clearly separated from the quasi-elastic line for $T_{S P} \lesssim T \lesssim J_{1}$. This discrepancy may be due to an overestimation of the temperature dependence of the spinon-stiffness in MF-theory [29,30].

Finally, I emphasize that the MF-theory gives only a limited description of spinon interaction effects. In the case of the two-spinon propagator this is known to result in an incorrect description of the spectral weight distribution [31]. This caveat of MF-theory has stimulated a study of approximate vertex corrections to the four-spinon-propagator [32]. However, the resulting Raman spectra show no agreement with experiment. At, present the impact of spinon interaction effects beyond MF-theory on the Raman spectra remain unclear.

In conclusion I have described a finite temperature MF-theory for frustrated spinchains and consequently detailed its application to magnetic Raman scattering. At lowtemperatures, in the gapless phase, I find a scattering continuum which is due to frustration induced four-spinon excitations and is compatible with observed Raman spectra of $\mathrm{CuGeO}_{3}$. In the high-temperature regime the MF Raman-intensity is dominated by a quasi-elastic line which results from the temperature dependence of the spinon spectrum. 


\section{ACKNOWLEDGMENTS}

I am indebted to B. Büchner, P. Knoll, and E. Müller-Hartmann for valuable discussions. Part of this work has been supported by the Deutsche Forschungsgemeinschaft through the SFB 341. 


\section{REFERENCES}

[1] M. Hase et al., Phys. Rev. Lett. 70, 3651 (1993).

[2] H. Kuroe et al., Phys. Rev. B 50, 16468 (1994).

[3] P.H.M. van Loosdrecht et al., Phys. Rev. Lett. 76, 311 (1996).

[4] P. Lemmens et al., Physica B 223\&224, 535 (1996).

[5] M. Nishi et al., Phys. Rev. B 50, 6508 (1994); M.C. Martin et al., Phys. Rev. B 53, R14713 (1996). L.P. Regnault et al., Physica B 213 \& 214, 278 (1995);

[6] J.P. Pouget et al., Phys. Rev. Lett. 72, 4037 (1994); O. Kamimura et al., J. Phys. Soc. Jpn. 63, 2467 (1994); S.B. Oseroff et al., Phys. Rev. Lett. 74, 1450 (1995).

[7] H. Völlenkle et al., Monatsh. Chem. 98, 1352 (1967); G.A. Petrakovskii et al., Zh. Eksp. Teor. Fiz. 98, 1382 (1990) [Sov. Phys. JETP 71, 772 (1990)]; K. Hirota et al., Phys. Rev. Lett. 73, 736 (1994); J.E. Lorenzo et al., Phys. Rev. B 50, 1278 (1994); Q.J. Harris et al., Phys. Rev. B 50, 12606 (1994); M. Arai et al., J. Phys. Soc. Jpn. 63, 1661 (1994).

[8] M. Braden et al., Phys. Rev. B 54, 1105 (1996).

[9] W. Geertsma and D. Khomskii, Phys. Rev. B 54, 3011 (1996).

[10] G. Castillia et al., Phys. Rev. Lett. 75, 1823 (1995).

[11] J. Riera et al., Phys. Rev. B 51, 16098 (1995).

[12] B. Büchner, unpublished.

[13] R. Chitra et al., Phys. Rev. B 52, 6581 (1995).

[14] M. Arai et al., Phys. Rev. Lett. 77, 3649 (1996).

[15] M. Aín et al., preprint.

[16] G.S. Uhrig and H.J. Schulz, Phys. Rev. B 54, R9624 (1996). 
[17] A. Fledderjohann and C. Gros, preprint cond-mat/9612013

[18] V.N. Muthukumar et al., Phys. Rev. B 54, R9635 (1996).

[19] P.H.M. van Loosdrecht et al., preprint cond-mat/9612167.

[20] C. Gros et al., preprint cond-mat/9612101.

[21] G.S. Uhrig, preprint, (1996).

[22] P. Jordan and E. Wigner, Z. Phys. 47, 631 (1928).

[23] L.N. Bulaevskii et al., Zh. Eksp. Teor. Fiz. 43, 968 (1962), [Sov. Phys. JETP 16, 685 $(1963)]$

[24] J. des Cloizeaux and J.J. Pearson, Phys. Rev. 128, 2131 (1962).

[25] T. Tonegawa and I. Harada, J. Phys. Soc. Jpn. 56, 2153 (1987).

[26] P.A. Fleury and R. Loudon, Phys. Rev. 166, 514 (1968).

[27] In Raman scattering photon-momentum transfer to the electronic system can be neglected which is implicit in the definition of $R$. Therefore spinon one-particle-hole excitations will contribute to the inelastic intensity only in case of a finite quasi-particle relaxation rate, which is absent in $\mathrm{MF}$-theory.

[28] G. Gómez-Santos, Phys. Rev. B 41, 6788 (1990).

[29] K. Frabricius et al., preprint cond-mat/9611077

[30] O. A. Starykh et al., preprint cond-mat/9701052.

[31] G. Müller, H. Beck, and J.C. Bonner, Phys. Rev. Lett. 43, 75 (1979).

[32] R.P.R. Singh et al., preprint (1996). 


\section{FIGURES}

FIG. 1. Temperature dependence of the mean-field hopping amplitude for various values of frustration.

FIG. 2. Mean-field Raman intensity (solid and dashed lines) at two temperatures and for

two values of frustration as compared to the experimental Raman spectrum of $\mathrm{CuGeO}_{3}$, [4, 18] (dashed-dotted line \& markers).

FIG. 3. Mean-field Raman intensity and susceptibility for various temperatures. 


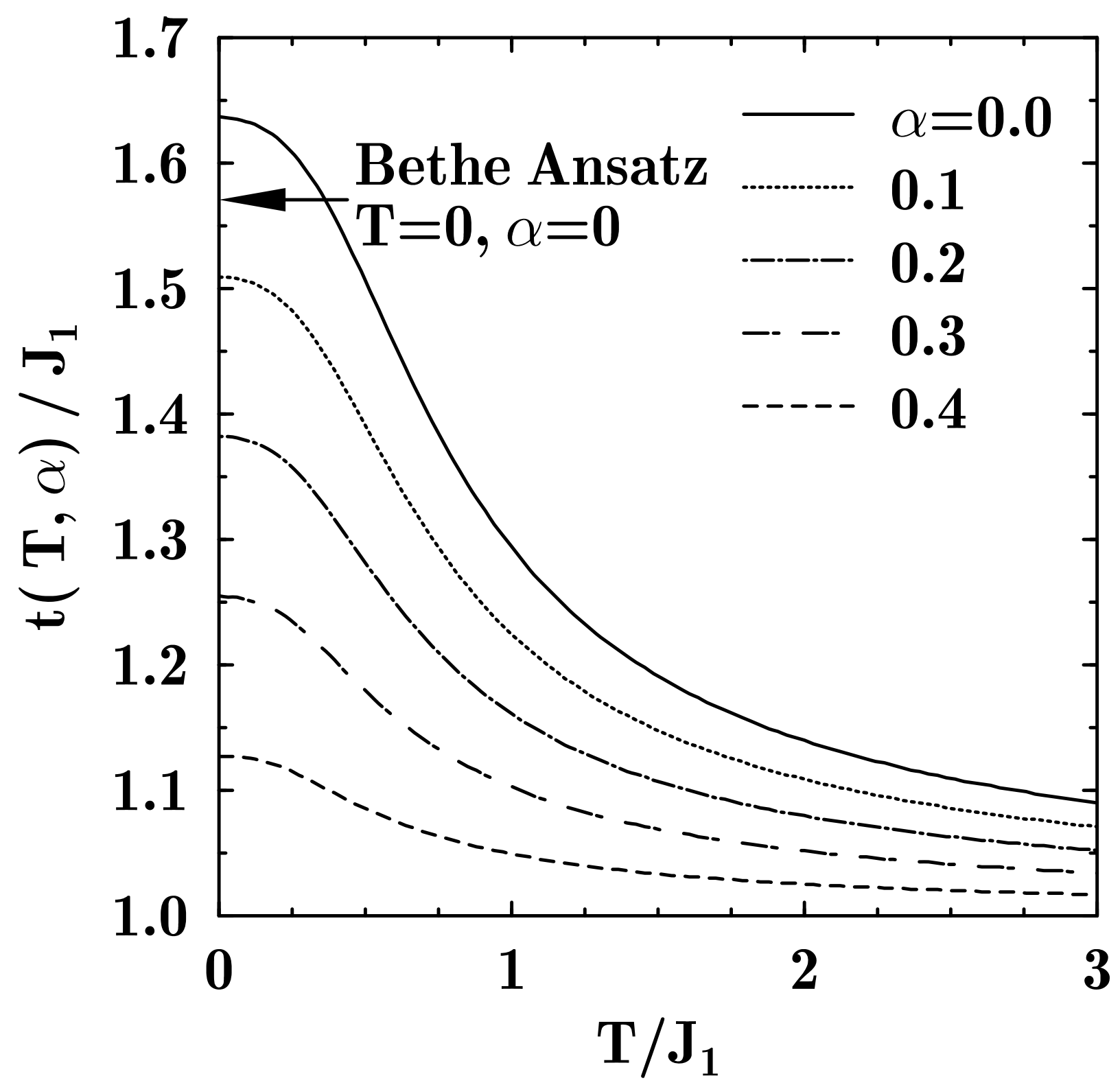

Fig. 1, Brenig 


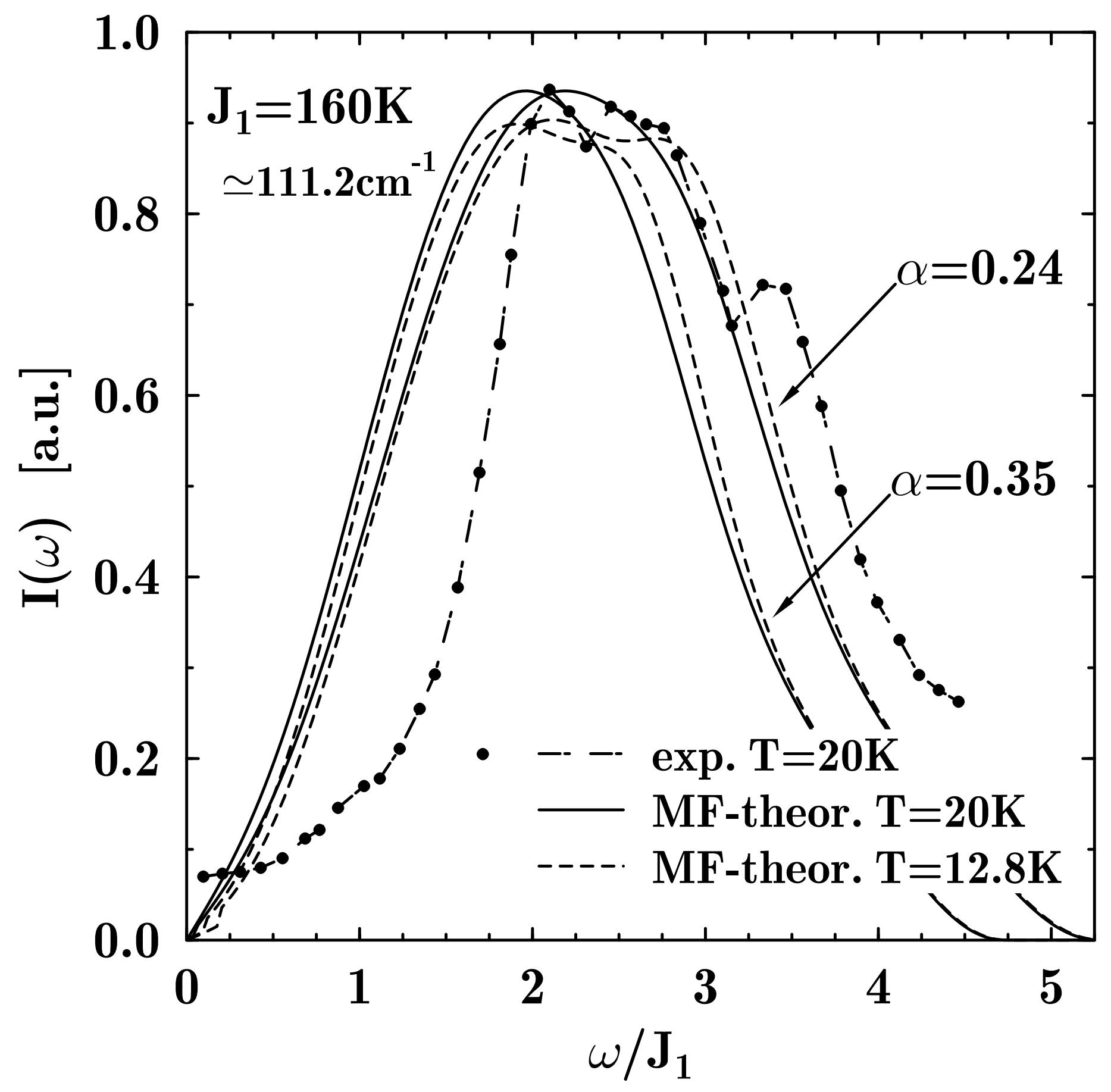

Fig. 2, Brenig 


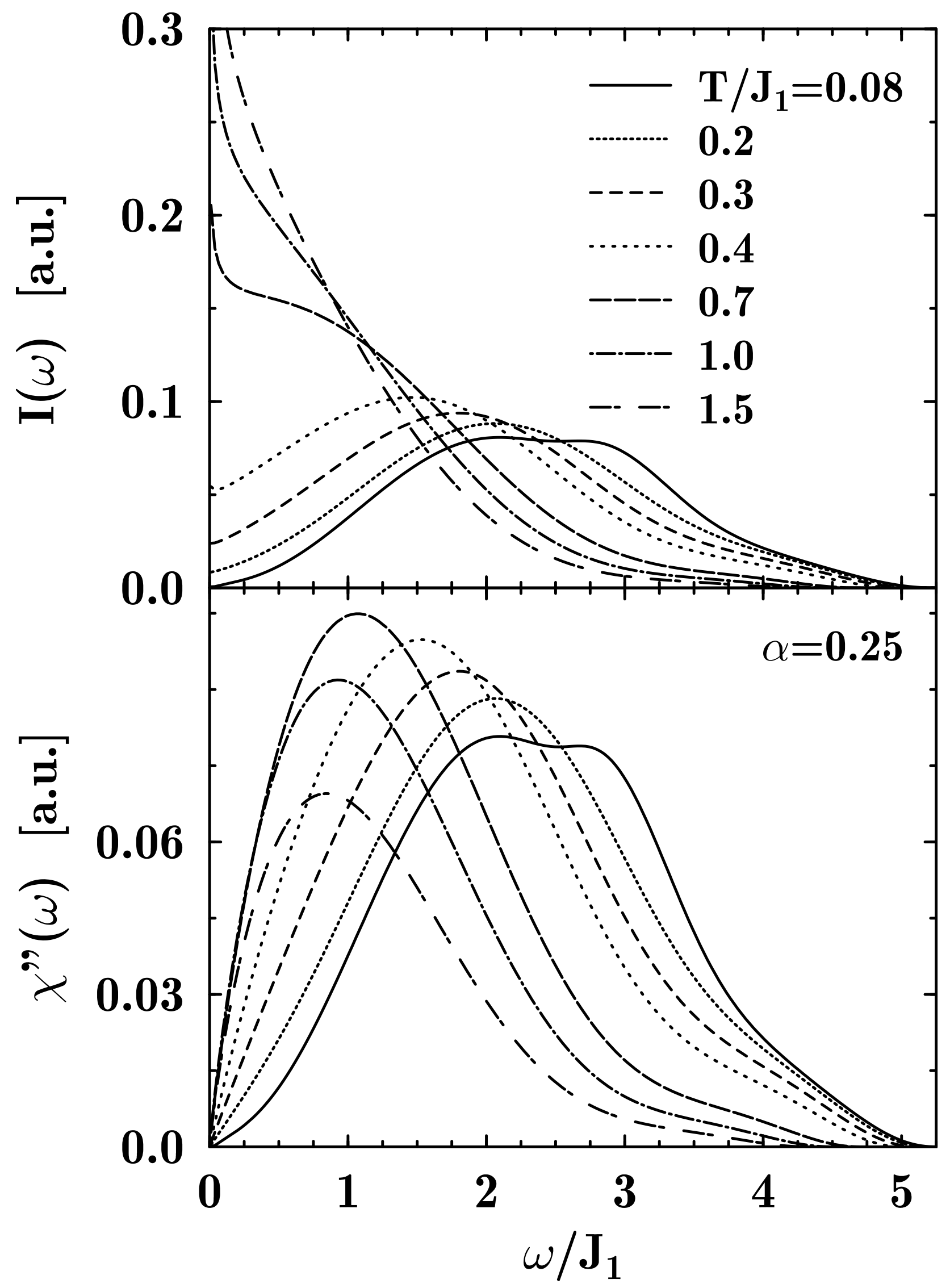

Fig. 3, Brenig 